\title{
Zur Rechtfertigung der Aggressionsmaschine
}

\author{
REINHARD HILKE \\ Fachbereich Philosophie, Geschichte und Sozialwissenschaften der Universität Erlangen-Nürnberg, \\ Institut für Psychologie
}

\section{WILHELM F. KeMPF}

Institut für die Pädagogik der Naturwissenschaften an der Universität KieI

\begin{abstract}
Es wird untersucht, in wieweit in Aggressionsmaschinen eine eindeutige Klassifikation von Handlungen als, ,aggressiv" bzw. ,nicht aggressiv" möglich ist. Ausgehend von einer Diskussion der konträren Definitionsvorschläge von BUSS und WERBIK wird ein Klassifikationssystem für ,,aggressive" Handlungen entwickelt, aus dem konkrete Hinweise für die Gestaltung von Versuchsanordnungen in der Aggressionsmaschine deduzierbar sind. Die Anwendbarkeit des Systems auf andere Versuchsanordnungen, wie sie in der Aggressions- und Konfliktforschung üblich sind, wird am Beispiel des „prisoner's dilemma game" aufgezeigt. Obwohl das Klassifikationssystem eine Weiterführung des WERBIKschen Definitionsvorschlages darstellt, kann auf ,,Sprachnormierung" verzichtet werden.
\end{abstract}

It is investigated whether the use of aggression machines yields an unambiguous classification of behavior as "aggressive" or ,non-aggressive". Starting with a discussion of the definitions suggested by BUSS and WERBIK a classification system for ,aggressive" behavior is developed. The system also applies to experimental situations outside the aggression machine. Concrete cues for experimental design are deduced from the system.

\section{Einleitung}

Seit die Aggressionsmaschine in den sechziger Jahren von Buss in die Aggressionsforschung eingeführt wurde, gilt sie weithin als die experimentelle Versuchsanordnung zur systematischen Erforschung aggressiven Verhaltens. Diese große Bedeutung, welche die Aggressionsmaschine für die Aggressionsforschung gewonnen hat, beruht auf der erreichten Standardisierung der Versuchssituationen einerseits und auf der scheinbaren Problemlosigkeit andererseits, mit der Handlungen in der Aggressionsmaschine als ,,aggressiv" bzw. ,,nicht aggressiv" klassifiziert werden können.

Definitions- und Klassifikationsprobleme sind in den letzten Jahren heftig diskutiert worden. In der vorliegenden Studie soll untersucht werden, inwieweit die Aggressionsmaschine die bezüglich dieser Problematik in sie gesetzten Hoffnungen erfüllen kann.
Die Aggressionsmaschine und die Aggressionsdefinition von Buss

Die Aggressionsmaschine von Buss (1961, p. $45 \mathrm{ff}$.) ist für zwei Personen konstruiert, für einen ,,Versuchsleiter" und eine ,,Versuchsperson". Dabei übernimmt die eigentliche Versuchsperson die Rolle des ,Versuchsleiters”, während die im Experiment als „Versuchsperson” eingeführte Person ein Gehilfe des richtigen Versuchsleiters ist. Der ,Versuchsleiter" wird dahingehend instruiert, daß es der Zweck der Untersuchung sei, den Einfluss des Geschlechtes und der Persönlichkeit des ,,Versuchsleiters" auf das Lernen von Konzepten festzustellen, und er die Aufgabe habe, die ,,Versuchsperson” immer dann einem Elektroschock auszusetzen, wenn sie im ,Lernexperiment" einen Fehler mache. Insgesamt stehen dem ,Versuchsleiter" an seinem Pult 10 Schockknöpfe zur Verfügung, d.h., er kann den Elektroschock in zehn verschiedenen Intensitätsgraden verabreichen. 
Damit der ,Versuchsleiter” eine Vorstellung von den verschiedenen Intensitäten hat, erhält er vor Beginn des Lernexperimentes Elektroschocks der Intensitätsstufen 1, 2, 3 und 5, und es wird ihm gesagt, daß die Stromstärke bei jeder Intensitätsstufe um den gleichen Betrag zunimmt. Während des Lernexperimentes gibt die „Versuchsperson" nach einem festgelegten Plan richtige und falsche Antworten und registriert, welche Intensitätsstufen der ,Versuchsleiter" gewählt hat. Die ,Versuchsperson” hat an ihrem Pult eine lichtdurchlässige Plastikscheibe, hinter der in Abhängigkeit von den gewählten Intensitätsstufen die Zahlen 1 bis 10 aufleuchten. Zwar ist die „Versuchsperson" an die Elektroden angeschlossen, der Stromkreis ist aber für die Dauer des Lernexperimentes unterbrochen.

\section{Wird die Busssche Aggressionsdefinition der Aggressionsmaschine gerecht?}

Einer objektiven Klassifikation von aggressiven Verhaltensweisen steht nach Ansicht von Buss (1961, p. 2 f.) hauptsächlich der introspektive Terminus ,,Intention” entgegen, welchen er demzufolge eliminiert, indem er untersucht, welche Charakteristika der jeweiligen Situation eine Verhaltensweise verstärken. Von dieser Uberlegung kommt er dann zu der Unterscheidung von ,,angry aggression" und ,,instrumentally aggressive responses", den zwei Hauptarten von Aggressionen, wobei die Klassifikation aufgrund der unterschiedlichen Verstärker erfolgt ${ }^{1}$.

Die objektive Feststellung eines Verstärkers setzt experimentell hergestellte und damit extrem standardisierte Situationen voraus. Denn die Feststellung eines Verstärkers ist nur möglich, wenn - unter Variation der potentiellen Verstärker - die Situation, in der die Verhaltensweise abläuft, mehrmals wiederholt werden kann. Durch die Konstruktion der Aggressionsmaschine wurde diesem Aspekt der Bussschen Aggressionsdefinition Rechnung getragen.

1 ,There are two major classes of reinforcers of aggression:

(1) the stimulus of the victim suffering injury or being in pain and

(2) extrinsic rewards." (Buss 1961, p.2.)
Zusätzlich führt Buss noch eine explizite Wertung der Verstärker ein, wenn er instrumentelle Aggressionen, sofern sie ein sozial akzeptiertes Ziel (reinforcer) anstreben, nicht als Aggression bezeichnet (vgl. Buss 1961, p. 3; 1971, p.9). Daß eine Klassifikation der Verstärker nach der sozialen Akzeptierung bzw. Nichtakzeptierung nicht ausreicht, zeigt sich aber gerade bei seinen Versuchen in der Aggressionsmaschine: Bei dem Nachweis, die Aggressionsmaschine erlaube eine eindeutige Klassifikation aggressiver Verhaltensweisen im Sinne seiner Aggressionsdefinition ${ }^{2}$, argumentiert er dahingehend, daß die Schocks der Intensitätsstufen 1 und 2 ,hinreichend mild" seien und demzufolge lediglich als ,Hinweise” (cues) anzusehen sind. Demgegenüber lägen die Schocks der Intensitätsstufen 3 bis 10 eindeutig über der Schmerzschwelle, sie seien damit zweifelsf rei aggressiv, da sie ,,noxious stimuli" liefern würden. Den möglichen Einwand, daß das Austeilen eines Elektroschocks nicht als aggressive Verhaltensweise klassifiziert werden kann, da der Versuchsleiter instruiert wurde, dies zu tun, entkräftet er wie folgt: ,When a subject delivers a painful stimulus in a situation that calls for only a cue, he is making an aggressive response; ...." (Buss 1961, p. 50).

Damit wertet er nicht nur die Verstärker, sondern die Relation Operation - Verstärker: Zwar ist das Lernen eines Konzeptes durch die Versuchsperson ein sozial akzeptierter Verstärker, aber Schocks der Intensitäten 3 bis 10 sind nicht das ,richtige" Mittel und deshalb agressiv. Das System von Bewertungsnormen bleibt Buss allerdings schuldig, und er rekurriert explizit auf sein Vorverständnis, wenn er schreibt: ,... und eine Bestrafung, die härter ist, als durch die gesellschaftliche Rolle zugelassen, muß bei ihrem wahren Namen genannt werden. Sie ist Aggression."'(Buss 1972, p. 27) 3

2 ,... aggression is defined as a response that delivers noxious stimuli to another organism." (Buss 1961, p. 1.)

${ }^{3}$ Es sei nur am Rande vermerkt, dass Buss seine Klassifikation selbst ad absurdum führt, wenn er Schocks der Intensitäten 1 und 2 als nicht aggressiv bezeichnet und jene der Intensitäten 3 bis 10 als aggressiv, und gleichzeitig den Mittelwert über alle der von einer Person gewählten Intensitätsstufen als Mass für deren Aggressivität verwendet. Zum Problem der Messung der Aggressivität siehe KEMPF (1974) und HıLKE et al. (1975). 
Der Rückgriff auf gesellschaftliche Rollen, d. h. auf den normativen Aspekt von Rollenerwartungen, der seine Definition - ohne ein System von Bewertungsnormen - von diesem Gesichtspunkt aus für Experimente in der Aggressionsmaschine untauglich werden läßt, scheint Buss dadurch gerechtfertigt zu sein, daß sein Klassifikationssystem auch im Alltag, d.h. bei freier Beobachtung, angewendet werden kann. Wir wollen deshalb der Frage nachgehen, ob dieser Anspruch haltbar ist.

\section{Die Eignung der Bussschen Aggressionsdefini- tion zur Klassifikation von Handlungen bei freier Beobachtung}

Wie wir bereits erläutert haben, sind Verstärker einer Verhaltensweise grundsätzlich nur in standardisierten Situationen objektiv feststellbar. Prinzipiell könnte damit das von Buss vorgeschlagene Klassifikationssystem bereits verworfen werden. Um aber die Probleme, die mit der Feststellung aggressiver Handlungen generell und im Alltag verbunden sind, möglichst deutlich zu machen, ist es notwendig, einige Zusatzannahmen von Buss zu diskutieren.

Buss lehnt ja einen,,intentionalen" Ansatz ab und muß deshalb angeben, wie zufällig gelieferte, schädliche Stimuli abgegrenzt werden können. Nach seiner Ansicht ist die Identifikation der Zufälligkeit bzw. Nichtzufälligkeit schädlicher Stimuli im Rahmen einer Zwei-Personen-Interaktionssituation (Aggressor - Opfer) anhand der ,,reinforcement history" der vom Aggressor gezeigten Reaktion möglich. Damit wäre aber jegliches Verhalten einer Person durch das ,Wissen”, das der Beobachter über frühere Verhaltensweisen dieser Person hat, determiniert und ein Verhalten außerhalb des Rahmens, der durch dieses ,Wissen” gesetzt wird, nicht möglich. Ungeachtet der faktisch vorliegenden Beweggründe einer Verhaltensweise würde nämlich der reine Ablauf vom Beobachter auf der Grundlage des ,Wissens” über frühere Abläufe gedeutet und auch diesem, ,Wissen" gemäss der jeweiligen Klasse von Verhaltensweisen zugeordnet. Schwerwiegender ist u. E. noch, daß eine Klassifikation von Verhaltensweisen aufgrund der ,reinforcement history" aus ethischen Gründen nicht vertretbar ist. Denn die
Klassifikation von Verhaltensweisen aufgrund der ,,reinforcement history” negiert die Möglichkeit der Veränderung habitueller Verhaltensweisen, indem die Konstanz habitueller Verhaltensweisen für die Klassifikation vorausgesetzt werden muß, und beraubt demzufolge den Einzelnen seiner ,,persönlichen Handlungsfreiheit". Hat eine Person eine aggressive ,,reinforcement history", so wird diese zur ,,selffulfilling prophecy": von der Person zufällig gelieferte (nicht intendierte), schädliche Stimuli sind als solche nicht identifizierbar, sondern vermehren nur das ,objektive Wissen" des Beobachters über die Aggressivität der Person. Über den deutschen Volksmund, Wer einmal lügt, dem glaubt man nicht, und wenn er auch die Wahrheit spricht" noch hinausgehend, wird die von einem Lügner ,,gesprochene Wahrheit" aufgrund seiner ,,reinforcement history" im Behaviorismus objektiv zur Lüge.

$\mathrm{Zu}$ einer weiteren Zusatzdefinition ist Buss gezwungen, weil der Begriff ,,Vorsatz" grundsätzlich nicht durch die Verstärker definiert werden kann: ,Intent is inferred by examining the stimuli antecedent to the response and its consequences." (Buss 1971, p. 10.) Nun kann die Behauptung, ein bestimmter Stimulus führe zu einer bestimmten Reaktion, entweder bedeuten, der Stimulus sei hinreichend oder er sei notwendig und hinreichend. Gehen wir von der ersten Behauptung aus, dann ist ein Stimulus nicht eindeutig einer Reaktion als ,Ursache” zuzuordnen. Wird behauptet, der Stimulus sei notwendig und hinreichend, so wird die deterministische Gesetzmässigkeit angenommen, daß eine Person immer dann und nur dann eine bestimmte Reaktion zeigt, wenn dieser Stimulus auftritt. Eine solche ,Gesetzmässigkeit" kann aber durch nahezu beliebige Daten widerlegt werden.

Dagegen könnte im Sinne von Buss eingewandt werden, daß die ,,reinforcement history" der von einer Person gezeigten Reaktion mit zu berücksichtigen sei. Ein bestimmter Stimulus könne nicht für beliebige Personen als ,Ursache” für eine bestimmte Reaktion betrachtet werden, sondern nur für jene Personen, für die die gleiche ,reinforcement history” einer Reaktion vorausgesetzt wird, d.h., ein Stimulus ist nur unter diesen Voraussetzungen notwendig und hinreichend für eine bestimmte Reaktion. In 
diesem Fall müßte aber der Nachweis erbracht werden, daß Personen mit identischer ,,reinforcement history" einer Reaktion existieren, ein Unterfangen, das a priori zum Scheitern verurteilt ist.

Am Beispiel der Bussschen Definition wird deutlich, daß ein Ansatz, der die ,Intention" nicht beinhaltet, nicht zu einer objektiven Klassifikation aggressiver Verhaltensweisen führen kann.

Ein System zur Klassifikation von ,,aggressiven" Handlungen nach der Intention

Ein wesentlicher Vorteil ,,intentionaler" Aggressionsdefinitionen ist, daß die objektive Klassifikation der faktischen Folgen einer Handlung nicht vorausgesetzt werden muß. Damit entfallen einige jener Probleme, die mit der Einführung des Begriffs ,,Schädigung", der in irgendeiner Art in jeder Aggressionsdefinition enthalten ist, verbunden sind ${ }^{4}$.

Bei intentionalen" Ansätzen besteht aber nun das Problem der Feststellbarkeit von Intentionen. Wie DANN (1972, p. 30) hervorhebt, zeichnen sich die Verfechter ,intentionaler" Ansätze dadurch aus, daß sie primär eine eindeutige Klassenbildung anstreben, während bei den Gegnern die objektive Feststellbarkeit im Vordergrund steht. Von den letzteren wird deshalb zu Recht kritisiert, daß die meisten Autoren, die ,intentionale" Definitionsversuche vorschlagen, dem Problem der Feststellbarkeit von Intentionen aus dem Wege gehen. Zwar scheinen sie an eine - wie auch immer geartete - Befragung der handelnden Person zu denken, sie kommen aber nicht, wie z. B. SchotT (1971) und WerbIK (1971, 1974), zu dem Schluß, daß einer Methode, die auf der Befragung der handelnden Person beruht, eindeutig der Vorzug zu geben sei. Im Gegensatz zu SchotT, der nur auf der Befragung der Person insistiert, gibt W'ERBIK eine konkrete Methode an.

\section{Das Klassifikationssystem von WERBIK}

\section{Das Klassifikationssystem von WERBIK ist ein}

\footnotetext{
${ }^{4} \mathrm{Vgl}$. HiLKE \& KEMPF (1973) und WeRBiK (1974).
}

Teil seiner kognitiven Theorie ,aggressiven” Handelns. In dieser Theorie wird den Aussagen der Person, die beobachtet wird, eine zentrale Stellung zugewiesen. ,Die Person wird vom Beobachter aufgefordert, auf Grundlage vorher getroffener sprachlicher Vereinbarungen ihren eigenen Handlungen Aussagen zuzuordnen. Das Verhalten einer Person wird durch ihre eigenen Aussagen klassifiziert." (WERBIK 1974, p. 141).

Die formalisierten Aussagen der handelnden Person (P1), die dieses Klassifikationssystem konstituieren, haben die erwarteten Wirkungen von Handlungen zum Gegenstand. WERBIK (1971) unterscheidet drei in diesem Kontext relevante Wirkungen (Zustandsveränderungen einer Person P2): die ,totale Zerstörung" (Tod) ${ }^{5}$, die „teilweise Zerstörung” (Verletzung) und den „unangenehmen inneren (aversiven) Zustand."

Die normierten Aussagen der handelnden Person (P1) lauten (nach WERBIK 1974, p. 150):

Kategorie von Handlungen Aussage von P1

\begin{tabular}{ll}
\hline $\begin{array}{l}\text { A. Destruktiv intendierte } \\
\text { Handlungen }\end{array}$ & $\begin{array}{l}\text {,Ich erwarte, daß } \\
\text { infolge meiner } \\
\text { Handlung der Tod } \\
\text { von P2 eintritt, und } \\
\text { ich handle.” }\end{array}$ \\
& ,Ich erwarte, daß \\
B. Teilweise destruktiv & Handlung eine Ver- \\
intendierte & letzung von P2 ein- \\
Handlungen & tritt, und ich \\
& handle." \\
& ,Ich erwarte, daß \\
& infolge meiner \\
Handlung ein aver- \\
siver Zustand für \\
Handlungen & P2 eintritt, und ich \\
& handle."
\end{tabular}

Wie wir bereits angedeutet haben, setzt das Klassifikationssystem von WERBIK voraus, daß ein normierter Sprachgebrauch mit der handelnden Person eingeübt wird, und daß der faktische Sprachgebrauch in der aktuellen Situation auch tatsächlich mit dem normierten Sprachge-

\footnotetext{
${ }^{5}$ Die in den Klammern angegebenen Begriffe werden bei WERBIK (1974) verwendet.
} 
brauch übereinstimmt. In diesem Fall ist die Verbalisierung von Absichten durch die handelnde Person für die Identifizierung von $\mathrm{Ab}$ sichten, und damit für die objektive Feststellbarkeit von , ,aggressiven" Handlungen, eine hinreichende Bedingung. WERBIK bezieht sich demzufolge von vornherein auf experimentelle Situationen. Denn einerseits ist es nur in normierten Situationen grundsätzlich möglich, eine Trainingsphase einzuführen, in der die Regeln für den Gebrauch der notwendigen Termini eingeübt werden, und andererseits setzt die Überprüfung, ob der faktische Sprachgebrauch mit dem normierten Sprachgebrauch übereinstimmt, experimentelle Anordnungen voraus.

Trotz des engen Geltungsbereiches wirft dieser Ansatz durch das Verfahren der Sprachnormierung und die Annahme, daß sich die Personen auch an die Sprachnormierung halten, eine Reihe von noch ungelösten Problemen auf und ist mit strengen Anforderungen an die Daten verbunden.

Problematisiert sei in diesem Kontext noch, daß, auch wenn die Annahme der Übereinstimmung von faktischem und normiertem Sprachgebrauch im Experiment einem Falsifikationsversuch standhält, die Annahme der generellen Übereinstimmung für die Dauer des gesamten Versuches die Erfahrung transzendiert.

Wir werden versuchen nachzuweisen, daß einerseits für die Klassifikation ,,aggressiver" Handlungen nach der Intention einer Person und andererseits für die Feststellung der Intention die strengen Annahmen von WERBIK nicht erforderlich sind. Insbesondere muß ein einheitlicher Sprachgebrauch bezüglich der erwarteten Wirkungen u.E. nur so weit hergestellt werden, daß die handelnde Person , ,bestimmte” Begriffe der Alltagssprache kennt.

\section{Der Sprachgebrauch über Wirkungen in seiner Bedeutung für die Erstellung eines Klassifikationssystems}

Gehen wir von den drei Kategorien von Wirkungen aus. wie sie WERBIK vorgeschlagen hat. so läßst sich zeigen, dar diese sich sowohl hinsichtlich der Möglichkeit, einen einheitlichen Sprachgebrauch herzustellen. als auch in Hirblick auf die faktische Einheitlichkeit des Sprachgebrauchs unterscheiden, was bei der Klassifikation der diese Wirkungen intendierenden Handlungen berücksichtigt werden muß.

Was den ,Zustand des Totseins” betrifft, kann der Sprachgebrauch als weitgehend vereinheitlicht angesehen werden, d.h., er ist mit Ausnahme von medizinisch-juristischen Grenzfällen eindeutig bestimmt, Insofern ist zwar eine eindeutige Prädikation auf der Basis von physikalischen Meßnormen (vgl. WERBIK 1974, p. 145) möglich, aber nicht notwendig.

Bezüglich ,,unangenehmer innerer (aversiver) Zustände" kann gemäß dem Vorschlag von WERBIK (1974, p. $145 \mathrm{ff}$.) ein einheitlicher Sprachgebrauch hergestellt werden: Ein $\mathrm{Zu}-$ stand einer Person wird dann als, unangenehmer innerer Zustand" bezeichnet, wenn die Person sich zur Beseitigung dieses Zustandes auffordert. Die Erwartung einer Person, daß infolge ihrer Handlung ein unangenehmer innerer Zustand einer (anderen) Person eintritt, ist dann gleichbedeutend mit der Erwartung, daß infolge der Handlung ein Zustand eintritt, zu dessen Beseitigung sich die andere Person auffordern wird. Mit dieser Herstellungsvorschrift ist der Nachteil verbunden, dał ,,negativ intendierte Handlungen" nur dann erklärt sind, wenn der Sprachgebrauch bereits vereinheitlicht ist. D. h. Handlungen sind nicht nur nicht als ,,negativ intendiert" feststellbar, sie können per definitionem der Klasse der negativ intendierten Handlungen gar nicht angehören, wenn die Person nicht die Verwendung bestimmter Termini wie ,Aufforderung”, ,Selbstaufforderung", "Ziel" usw. gelernt hat, um die entsprechende Erwartung auszubilden.

Eine Vereinheitlichung des Sprachgebrauchs bezüglich ,unangenehmer innerer Zustände" in dem Sinne, dak eine bestimmte Teilmenge von Zuständen durch alle Mitglieder einer definierten Gruppe in gleicher Weise als , unangenehme innere Zustände" kategorisiert wird, ist aber für eine objektive Klassifikation von Handlungen als ,negativ intendiert" keine notwendige Voraussetzung. Denn die Erwartung der handelnden Person ist ein ,,private event", für dessen Inhalt der Sprachgebrauch der handelnden Person makgebend ist. Im Rahmen dieses Sprachgebrauchs mufi die handelnde Person lediglich ein alltagssprachliches Vorverständnis 
von ,,unangenehmen inneren Zuständen" haben

Nicht als vereinheitlicht kann der Sprachgebrauch über den Zustand der ,,teilweisen Zerstörung" (Verletzung) angesehen werden. WERBIK (1974, p. 146f.) ist der Ansicht, daß über eine einfache Klassifikation des Organismus eine Vereinheitlichung des Sprachgebrauchs zu erreichen ist, wobei dieser Sprachgebrauch zwangsläufig an dem der Experten orientiert sein muss. Der Rekurs auf ein „Expertenurteil” verschiebt aber nur das Problem, denn auch „teilweise destruktiv intendierte Handlungen" sind dann generell nicht erklärt, wenn nicht alle Personen dasselbe System zur Klassifikation des Organismus verwenden. Ein ,,einheitlicher" Sprachgebrauch, dem der der Experten zugrundeliegt, kann faktisch nicht hergestellt werden, da dazu - zumindest im Bereich der Humanwissenschaften - der Zustand ,,letztmöglicher Erkenntnis" erreicht sein müßte.

Wessen Sprachgebrauch soll nun aber für die Klassifikation maßgebend sein, der der handelnden Person oder der der betroffenen Person, wenn von einem einheitlichen Sprachgebrauch nicht ausgegangen werden kann? Es ist ja durchaus möglich, daß eine Person einen infolge der von ihr ausgeführten Operation bei einer anderen Person eingetretenen Zustand nicht als ,,teilweise Zerstörung" klassifiziert, während ihn die betroffene Person als solchen klassifiziert. So wird ein Arzt, der bei einem Patienten die Akupunktur anwendet, den $\mathrm{Zu}$ stand des Patienten nicht als, ,Verletzung" klassifizieren, während der Patient, der die Akupunktur nicht kennt und demzufolge für eine Foltermethode hält, dies tut.

Es ist nun auch der umgekehrte Fall denkbar: Wenn einem Forscher, der studienhalber in der Manus-Gesellschaft lebt, die ,Ehre" zuteil wird, im Rahmen des dafür veranstalteten Festes einem Manus-Knaben beide Ohren mit einem zugespitzten Holz zu durchstechen und die Löcher auszuweiten (vgl. MEAD 1965, p. 274), so wird er den Zustand des Knaben als ,Verletzung” klassifizieren. Für den Knaben ist dieses Zeichen der ,Mannhaftigkeit" sicher keine Verletzung.

Aus den genannten Gründen ist es notwendig, den möglicherweise unterschiedlichen Sprachgebrauch bei der Klassenbildung zu berücksichtigen, und wir schlagen gemäß dem intentionalen Ansatz vor, die Klassifikation ,,teilweise de- struktiv intendierter Handlungen" sowohl auf der Grundlage des Sprachgebrauchs der handelnden Person als auch auf der Grundlage des ,Wissens" der handelnden Person über den Sprachgebrauch der betroffenen Person vorzunehmen. Besondere Relevanz hat dieser Ansatz für die Erforschung aggressiven Verhaltens bei Kindern.

Bei der Klassifikation von Handlungen als ,negativ intendiert" ergibt sich ein analoges Problem nicht, denn im Gegensatz zur ,,teilweisen Zerstörung" ist der ,,unangenehme innere Zustand" der betroffenen Person deren ,,private event". Für die Klassifikation einer Handlung als ,negativ intendiert" ist daher ausschließlich das „Wissen" der handelnden Person über die Klassifikation des Zustandes durch die betroffene Person relevant.

\section{Das Klassifikationssystem}

Das folgende Klassifikationssystem für intendierte Handlungen wurde unter expliziter Bezugnahme auf die kognitive Terminologie von WERBIK \& KEMPF (1972) entwickelt. Dieses terminologische System enthält Aussagen über kognitive Vorgänge (Selbstaufforderungen, Erwartungen usw.). Die Aussagen sind theoriesprachlich, d.h., ihre Bedeutung ist ausschließlich durch bestimmte, explizit vereinbarte Prädikatorenregeln festgelegt.

Anders als bei WeRBIK $(1971,1974)$ werden die kognitiven Vorgänge nicht als Aussagen der Person formuliert, sondern lediglich Regeln darüber festgelegt, wie der Sprachgebrauch der Beobachter bei Aussagen über die Probanden $\mathbf{z u}$ vereinheitlichen ist. Bei dieser Interpretation des terminologischen Systems wird somit z. B. die Handlung einer Person nicht mehr dann und nur dann als ,,destruktiv intendiert" klassifiziert, wenn die handelnde Person der Aussage: ,Ich erwarte, daß infolge meiner Handlung der Tod von P2 eintritt', zustimmt, sondern dann, wenn der Beobachter aufgrund der Sprachregeln die Aussage: „Die Person erwartet, daß infolge ihrer Handlung der Tod von P2 eintritt", treffen kann.

In dem von WERBIK \& KEMPF (1972) vorgeschlagenen terminologischen System wird jede Handlung einer Person als hierarchisches System von ,Selbstaufforderungen" verstanden. Hat sich eine Person zur Erreichung 
eines Zieles $z_{0}$ aufgefordert und tritt dieses Ziel nicht inzidentell ein, so kann die Person entweder die Selbstaufforderung $z_{0}$ ! wieder zurücknehmen, d.h. auf die Erreichung von $z_{0}$ verzichten, oder sich zur Erreichung eines Zieles $z_{1}$ aufforder $n$, von dem sie erwartet, daß es für die Erreichung des Zieles $z_{0}$ ein geeignetes Mittel ist. Das Ziel $z_{1}$ heißt ein Unterziel des Zieles $z_{0}, z_{0}$ ein Oberziel des Zieles $z_{1}$. Grundsätzlich kann jedes Ziel, zu dessen Erreichung sich eine Person auffordert, als Unterziel zu einem übergeordneten Ziel verstanden werden. Da eine Operation durch das ihr zugeordnete Ziel eindeutig bestimmt ist, sind die Termini „Operation" und „Ziel" extensionsgleich und können synony $m$ verwendet werden.

Die Ausführung einer Operation $z_{0}$ wird als Selbstaufforderung zur Erreichung des Zieles $z_{0}$ und zur Erreichung eines, dem Ziel $z_{0}$ untergeordneten, Zieles $z_{j}$ auf $j$ edem tiefergelegenen Niveau $j=1,2,3, \ldots$ der hierarchischen Organisation beschrieben:

$z_{0} ! \wedge \wedge_{j} \bigvee z_{j}: z_{j} !$, kurz , , $z_{0} !$ und ich handle".

Hat sich eine Person zur Erreichung eines Zieles $z_{0}$ aufgefordert, so ist die Erwartung , , $z_{1}$ ist für $z_{0}$ hinreichend"

$e\left(z_{1} \rightarrow z_{0}\right)$

notwendige Voraussetzung für die Selbstaufforderung $z_{1}$ !

Im Rahmen der vorliegenden Arbeit - insbesondere wegen der Frage nach der Feststellbarkeit von Intentionen - wird es rotwendig, das terminologische System um die folgenden Definitionen und Prädikatorenregeln zu erweitern:

\section{Definition 1:}

„Eine Person $P_{v}$ weiß ${ }^{6}$, daß die Ausführung einer Operation $z_{0}$ für das Eintreten eines Ereignisses $c$ hinreichend ist, genau dann, wenn das Eintreten des Ereignisses $\mathrm{c}$ in dem Ziel $z_{0}$ als ,Teilziel' enthalten ist."

$(D-1): w\left(z_{0} \rightarrow c\right) \Leftrightarrow c \epsilon z_{0}$

\section{Prädikatorenregel 1:}

,Hat sich eine Person $\mathbf{P}_{\mathbf{v}}$ zur Erreichung eines Zieles $\mathbf{z}_{\mathbf{0}}$ aufgefordert und ist das Eintreten eines Ereignisses $\mathrm{c}$ in dem Ziel enthalten, dann ist erlaubt zu sagen, die Person $P_{v}$ habe sich (unter anderem) zur Erreichung des Teilzieles c aufgefordert."

$(P-1):\left(z_{0} ! \wedge w\left(z_{0} \rightarrow c\right)\right) \Rightarrow c !$

\section{Prädikatorenregel 2:}

„Hat eine Person die Erwartung $e\left(z_{1} \rightarrow z_{0}\right)$ und ist die

${ }^{6}$ Mit der Teilaussage ,eine Person $P_{v}$ weilf" ist nicht impliziert, daß der gewulste Inhalt auch objektiv richtig ist.
Herstellung eines Ereignisses $c$ als Teilziel in $z_{0}$ enthalten, dann ist erlaubt zu sagen, die Person hat die Erwartung $\mathrm{e}\left(\mathrm{z}_{1} \rightarrow \mathrm{c}\right)$."

$$
(P-2):\left(e\left(z_{1} \rightarrow z_{0}\right) \wedge w\left(z_{0} \rightarrow c\right)\right) \Rightarrow e\left(z_{1} \rightarrow c\right)
$$

\section{Definition 2:}

„Die handelnde Person $P_{\mathrm{v}}$ erwartet, daß die Ausführung

$z_{0} ! \wedge \wedge_{j} \vee z_{j}: z_{j} !$

der Operation $z_{0}$ für das Eintreten eines Ereignisses c hinreichend ist, genau dann, wenn die Person $P_{v}$ erwartet, daß $z_{0}$ für das Eintreten von $c$ hinreichend ist $\left(e\left(z_{0} \rightarrow c\right)\right)$, und/oder wenn die Ausführung der Operation $z_{0}$ mit Notwendigkeit die Selbstaufforderung zur Erreichung eines Zieles $z_{j}=z_{1}, z_{2}, z_{3}, \ldots$ einschließt, von welchem die Person $P_{v}$ erwartet, daß $z_{j}$ für das Eintreten von $c$ hinreichend ist."

$$
\begin{aligned}
(D-2): & e\left(\left(z_{0} ! \wedge \bigwedge_{j} \vee z_{j}: z_{j} !\right) \rightarrow c\right) \Leftrightarrow e\left(z_{0} \rightarrow c\right) \vee \\
& \left(\left(z_{0} ! \wedge \bigwedge_{j} z_{j}: z_{j} !\right) \supset\left(\bigvee z_{j}: z_{j} ! \wedge e\left(z_{j} \rightarrow c\right)\right)\right)
\end{aligned}
$$

Für die Anwendung dieser Definitionen und Prädikatorenregeln muß auch noch die empirische Voraussetzung gefordert werden, wonach eine Person $P_{v}$ ein ,Wissen" darüber erwerben kann bzw. erworben hat, daß das Eintreten eines Ereignisses $c$ in einem Ziel $z_{0}$ enthalten ist.

Auf der Grundlage dieser Voraussetzungen schlagen wir vor, Handlungen der Form , $z_{0}$ ! und ich handle" wie folgt nach der Intention der handelnden Person zu klassifizieren:

Eine Handlung , , $\mathrm{z}_{0}$ ! und ich handle" heißt $(\mathrm{K}-1)$ :

Destruktiv-intendiert, wenn die handelnde Person P1 erwartet, daß die Ausführung der Operation $\mathrm{z}_{0}$ hinreichend ist für den Tod einer (anderen) Person P2.

$(K-2)$ :

Teilweise-destruktiv-intendiert, wenn die handelnde Person P1 erwartet, daß die Ausführung der Operation $z_{0}$ hinreichend ist für die Herstellung eines Zustandes bei einer (anderen) Person $\mathrm{P} 2$, von dem P1 weih, daß er eine Verletzung ist, und/oder von dem sie weiß, daß ihn die Person P2 als Verletzung klassifiziert.

$(\mathrm{K}-2.1)$ :

Einfach teilweise-destruktiv-intendiert, wenn 
die handelnde Person P1 erwartet, dafs die Ausführung der Operation $z_{0}$ hinreichend ist für die Herstellung eines Zustandes bei einer (anderen) Person P2, von dem P1 weik, daks er eine Verletzung ist, von dem sie aber nicht weils, wie ihn die Person P2 klassifiziert.

$(\mathrm{K}-2.2)$ :

Offenkundig teilweise-destruktiv-intendiert, wenn die handelnde Person P1 erwartet, daß die Ausführung der Operation $z_{0}$ hinreichend ist für die Herstellung eines Zustandes bei einer (anderen) Person P2, von dem P1 weiß, daß er eine Verletzung ist, und von dem sie weiß, daß ihn die Person P2 als Verletzung klassifiziert.

$(\mathrm{K}-2.3)$ :

Verdeckt teilweise-ilestruktiv-intendiert, wenn die handelnde Person P1 erwartet, dał die Ausführung der Operation $z_{0}$ hinreichend ist für die Herstellung eines Zustandes bei einer (anderen) Person P2, von dem P1 weiß, dab er eine Verletzung ist, von dem sie aber weiß, daß ihn die Person P2 nicht als Verletzung klassifiziert.

$(\mathrm{K}-2.4)$ :

Disparat teilweise-destruktiv-intendiert, wenn die handelnde Person P1 erwartet, dab die Ausführung der Operation $\mathrm{z}_{0}$ hinreichend ist für die Herstellung eines Zustandes bei einer (anderen) Person P2, von dem P1 weiß, dah er keine Verletzung ist, von dem sie aber weib, daß ihn die Person P2 als Verletzung klassifiziert.

$(\mathrm{K}-3)$ :

Negativ intendiert, wenn die handelnde Person P1 erwartet, daß die Ausführung der Operation $\mathrm{z}_{0}$ hinreichend ist für die Herstellung eines $\mathrm{Zu}$ standes bei einer (anderen) Person P2, von dem $P 1$ weiß, daß ihn die Person P2 als unangenehmen inneren Zustand klassifiziert.

(K-4):

Nicht destruktiv-intendiert, wenn die handelnde Person P1 nicht erwartet, dafs die Ausführung der Operation $z_{0}$ hinreichend ist für den Tod einer (anderen) Person P2.

$(\mathrm{K}-5)$ :

Nicht teilweise-destruktiv-intendiert, wenn die handelucie Person P1 nicht erwartet. dafi die
Ausführung der Operation $z_{0}$ hinreichend ist für die Herstellung eines Zustandes bei einer (anderen) Person P2, von dem P1 weiß, daß er eine Verletzung ist, und/oder, von dem sie weiß, daki ihn die Person P2 als Verletzung klassifiziert.

$(\mathrm{K}-6)$ :

Nicht negativ intendiert, wenn die handelnde Person P1 nicht erwartet, daß die Ausführung der Operation $\mathrm{z}_{0}$ hinreichend ist für die Herstellung eines Zustandes bei einer (anderen) Person $P 2$, von dem $P 1$ weiß, daß ihn die Person P2 als unangenehmen inneren Zustand klassifiziert.

Die bisherigen Überlegungen haben sich stets auf die Prädikation ausgeführter Handlungen bezogen. Nun mus aber auch der Verzicht auf die Erreichung eines Zieles $z_{0}\left(\neg\left(z_{0}\right.\right.$ !) ebenfalls als eine Handlungsalternative angesehen werden, was zur Folge hat, daß auch der Verzicht auf die Erreichung eines Zieles $z_{0}$ als ,aggressive Handlung" klassifiziert werden muß, wenn die handelnde Person P1 erwartet, daß die Nichterreichung des Zieles $z_{0}$ für eine andere Person $P 2$ einen unangenehmen inneren Zustand, eine teilweise oder eine totale Zerstörung bewirkt.

Diese Definition ist aber nur brauchbar, wenn zuvor geklärt wurde, was die Rede von ,,erwarteten Wirkungen" eines Verzichtes bedeutet; bei WERBIK \& KEMPF (1972) ist die Erwartung, daß der Verzicht auf die Erreichung eines Zieles $z_{0}$ für die Herstellung eines Ereignisses $c$ hinreichend ist, $\left(\mathrm{e}\left(\neg\left(\mathrm{z}_{0} !\right) \rightarrow \mathrm{c}\right)\right)$ nicht erklärt.

HIL KE \& KEмPF (1973) schlagen deshalb vor, die Erwartung ef $\left.\neg\left(z_{0} !\right) \rightarrow c\right)$ über die Definition 3

$$
\begin{gathered}
(D-3): w\left(z_{0} \rightarrow \neg c\right) \wedge \neg\left(z_{0} !\right) \wedge \neg \vee z_{0}^{\prime}: w\left(z_{0}^{\prime} \rightarrow \neg c\right) \wedge z_{0}^{\prime} ! \Leftrightarrow \\
e\left(\neg\left(z_{0} !\right) \rightarrow c\right)
\end{gathered}
$$

einzuführen: ,Die handelnde Person $P_{v}$ erwartet genau dann, daß der Verzicht auf die Erreichung des Zieles $z_{0}$ für das Eintreten eines Ereignisses c hinreichend ist, wenn die Person $P_{v}$ weiß, daß die Erreichung des Zieles $z_{0}$ für das Eintreten von $7 c$ (d.h. für die Beseitigung bzw. die Verhinderung des Ereignisses c) hinreichend ist, und auf die Erreichung von $\mathrm{z}_{0}$ verzichtet, und es kein anderes $Z_{\text {iel }} z_{0}$ gibt, von dem die Person $P_{\mathbf{v}}$ weib, daß die Erreichung dieses Zieles $z_{0}^{\prime}$ für das Eintreten von $7 c$ hinreichend ist, und zu dessen Erreichung sich die Person auffordert."

Auf der Grundlage dieser Definition ergibt sich dann die folgende Erweiterung des Klassifikationssystems $(K-1)$ bis $(K-6)$ :

Der Verzicht $7\left(\mathrm{z}_{0}\right.$ !) auf die Erreichung eines Zieles $z_{0}$ heilit 
$\left(\mathrm{K}-1^{*}\right)$ :

Destruktiv-intendiert, wenn die handelnde Person P1 erwartet, dał der Verzicht $\neg\left(z_{0}\right.$ !) auf die Erreichung des Zieles $z_{0}$ hinreichend ist für den Tod einer (anderen) Person P2.

$\left(\mathrm{K}-2^{*}\right)$ :

Teilweise-destruktiv-intendiert, wenn die handelnde Person P1 erwartet, daß der Verzicht $\neg\left(z_{0}\right.$ !) auf die Erreichung des Zieles $z_{0}$ hinreichend ist für die Herstellung eines Zustandes bei einer (anderen) Person P2, von dem P1 weiß , daß er eine Verletzung ist, und/oder von dem sie weiß, daks ihn die Person P2 als Verletzung klassifiziert.

$\left(\mathrm{K}-2.1^{*}\right)$ :

Einfach-teilweise-destruktiv-intendiert, wenn die handelnde Person P1 erwartet, daß der Verzicht $\checkmark\left(z_{0}\right.$ !) auf die Erreichung des Zieles $z_{0}$ hinreichend ist für die Herstellung eines Zustandes bei einer (anderen) Person P2, von dem P1 weith, dath er eine Verletzung ist, von dem sie aber nicht weiß, wie ihn die Person P2 klassifiziert.

$(\mathrm{K}-2.2 *)$ :

Offenkundig teilweise-destruktiv-intendiert, wenn die handelnde Person $\mathbf{P} 1$ erwartet, daß der Verzicht $\neg\left(z_{0}\right.$ !) auf die Erreichung des Zieles $z_{0}$ hinreichend ist für die Herstellung eines Zustandes bei einer (anderen) Person P2, von dem $P 1$ weißs, daß3 er eine Verletzung ist, und von dem sie weik, dał ihn die Person P2 als Verletzung klassifiziert.

$\left(K-2.3^{*}\right)$ :

Verdeckt-teilweise-destruktil-intendiert, wenn die handelnde Person P 1 erwartet, daß der Verzicht $\neg\left(z_{0}\right.$ !) auf die Erreichung des Zieles $z_{0}$ hinreichend ist für die Herstellung eines Zustandes bei einer (anderen) Person P2, von dem P1 weiß, dak er eine Verletzung ist, von dem sie aber weils, dals ihn die Person P2 nicht als Verletzung klassifiziert.

$\left(\mathrm{K}-2.4^{*}\right)$ :

Disparat teilweise-destruktiv-intendiert, wenn die handelnde Person Pl erwartet. dals der Verzicht $\urcorner\left(z_{0}\right.$ !) auf die Erreichung des Zieles $z_{0}$ hinreichend ist für die Herstellung eines Zustandes bei einer (anderen) Person P2, von dem sie weiß, daß er keine Verletzung ist, von dem sie aber weiß, daß ihn die Person $\mathrm{P} 2$ als Verletzung klassifiziert.

$(\mathrm{K}-3 *)$ :

Negativ intendiert: wenn die handelnde Person P1 erwartet, daß der Verzicht $\neg\left(z_{0}\right.$ !) auf die Erreichung des Zieles $z_{0}$ hinreichend ist für die Herstellung eines Zustandes bei einer (anderen) Person P2, von dem P1 weiß, daß ihn die Person $\mathrm{P} 2$ als unangenehmen inneren Zustand klassifiziert.

$\left(\mathrm{K}-4^{*}\right)$ :

Nicht-destruktiv-intendiert, wenn die handelnde Person $\mathrm{P} 1$ nicht erwartet, daß3 der Verzicht \urcorner$\left(z_{0} !\right)$ auf die Erreichung eines Zieles $z_{0}$ hinreichend ist für den Tod einer (anderen) Person P2.

\section{$\left(\mathrm{K}-5^{*}\right)$ :}

Nicht teilweise-destruktiv-intendiert, wenn die handelnde Person P1 nicht erwartet, daß der Verzicht $\neg\left(z_{0}\right.$ !) auf die Erreichung eines Zieles $\mathrm{z}_{0}$ hinreichend ist für die Herstellung eines $\mathrm{Zu}$ standes bei einer (anderen) Person P2, von dem $\mathrm{P} 1$ weifh, daß3 er eine Verletzung ist, und/oder von dem sie weiß, daß ihn die Person P2 als Verletzung klassifiziert.

$\left(K-6^{*}\right)$ :

Nicht negativ-intendiert, wenn die handelnde Person P1 nicht erwartet, daß der Verzicht $\neg\left(z_{0}\right.$ !) auf die Erreichung des Zieles $z_{0}$ hinreichend ist für die Herstellung eines Zustandes bei einer (anderen) Person P2, von dem P1 weiß, dał ihn die Person $P 2$ als unangenehmen inneren Zustand klassifiziert.

\section{Zur Feststellbarkeit ,aggressiver” Handlungen in der Aggressionsmaschine}

Wie wir gesehen haben, sind für die Feststellung ,aggressiver" Handlungen nach dem Klassifikationssystem von WERBIK einerseits die Sprachnormierung als Verfahren und andererseits die Übereinstimmung von faktischem und normiertem Sprachgebrauch erforderlich. 
Ist die auf Seite 53 getroffene empirische Voraussetzung erfüllt, d.h., hat die Person $\mathrm{P}_{v}$ ein „Wissen” über Teilzielrelationen erworben, dann ermöglicht das von uns vorgeschlagene System ebenfalls die Klassifikation von aktuellen Handlungen aufgrund der intendierten Wirkungen. Denn aus den terminologischen Vereinbarungen $D-2$ und $P-2$ kann gefolgert werden, eine Person $\mathrm{P}_{\mathrm{v}}$ erwarte, daß die Ausführung einer Handlung , , $\mathrm{z}_{0}$ ! und ich handle" für das Eintreten eines Ereignisses $c$ hinreichend ist, wenn die Person weiß, daß $\mathrm{z}_{0}$ für $\mathrm{c}$ hinreichend ist:

$w\left(z_{0} \rightarrow c\right) \operatorname{De}\left(\left(z_{0} ! \wedge \bigwedge_{j} \vee z_{j}: z_{j} !\right) \rightarrow c\right)$

Beweis:

Aus $\mathrm{P}-2$ folgt:

(1) $w\left(z_{0} \rightarrow c\right) \supset\left(\wedge z_{1}: e\left(z_{1} \rightarrow z_{0}\right) \supset e\left(z_{1} \rightarrow c\right)\right)$.

Aufgrund der Definition , $z_{0}$ ! und ich handle" =:

$z_{0} ! \wedge \bigwedge_{j} \bar{z}_{j}: z_{j} !$ folgt:

(2) , $z_{0} !$ und ich handle" $\supset \bigvee z_{1}: z_{1} !$

Da aber nach KEMPF (1973, p. 52):

(3) $\mathrm{z}_{1} ! \Rightarrow \mathrm{e}\left(\mathrm{z}_{1} \rightarrow \mathrm{z}_{0}\right)$

folgt:

(4) „, $z_{0}$ ! und ich handle" $\supset\left(\bigvee z_{1}: z_{1} ! \wedge e\left(z_{1} \rightarrow z_{0}\right)\right)$

Unter Verwendung von (1) folgt schließlich:

(5) $w\left(z_{0} \rightarrow c\right) \supset\left(, z_{0}\right.$ ! und ich handle" $\supset\left(\bigvee z_{1}\right.$ : $\left.\left.z_{1} ! \wedge e\left(z_{1} \rightarrow c\right)\right)\right)$

q.e.d.

Gehen wir von der Annahme aus, daß Personen das „Wissen” über Teilzielrelationen durch Lernen erwerben können, so ist dieser Ansatz eng mit der experimentalpsychologischen Methodik verknüpft. Denn nur das Experiment gestattet es, Personen mit Handlungsmöglichkeiten zu konfrontieren, über die sie kein aus dem Alltag stammendes ,Vorwissen” haben, oder das , Vorwissen” über diese Handlungsmöglichkeiten zu kontrollieren. Weiterhin müssen die Personen lernen können, manche Handlungsalternativen seien für bestimmte Zustandsveränderungen einer anderen Person immer hin- reichend (Teilziel $c$ ist in Ziel $z_{0}$ enthalten) und andere immer nicht (Teilziel $c$ ist in Ziel $z_{0}$ nicht enthalten).

Um sicherzustellen, daß sowohl die Handlungsmöglichkeiten als auch die Wirkungen dieser Handlungen über den gesamten Versuch konstant sind, ist die Verwendung einer Apparatur (Aggressionsmaschine) eine geeignete Bedingung. Im Gegensatz zur Aggressionsmaschine von Buss müßten der $\mathrm{Vp}$ in einer solchen Apparatur aber mindestens eine ,,aggressive" und eine ,neutrale" Handlung zur Verfügung stehen, d.h. eine Handlung, die immer zu einer bestimmten Zustandsveränderung einer anderen Person führt, und eine Handlung, die nie zu dieser bestimmten Zustandsveränderung einer anderen Person führt. Dies muß unabhängig davon gelten, welche anderen Teilziele $\mathrm{c}$ bei der jeweiligen Handlung in dem Ziel $z_{0}$ enthalten sind.

Aus ethischen und moralischen Gründen kann nur die experimentelle Realisierung von ,unangenehmen inneren Zuständen" vertreten werden, wobei die Verwendung bestimmter Teilklassen der Klasse von ,unangenehmen inneren Zuständen" sicherlich ebenfalls ausgeschlossen werden muß.

Da die konkrete Gestaltung der Lernphase wesentlich durch die jeweilige Fragestellung determiniert ist, wollen wir dieses Thema nur soweit diskutieren, wie es für den Ansatz von genereller Bedeutung ist. Zwar ist in experimentellen Situationen die Annahme, eine Person habe das relevante Wissen durch Lernen erworben, grundsätzlich nur falsifizierbar, die Annahme, daß sich das Wissen während der Versuchsdurchführung nicht ändert, transzendiert aber ebenfalls die Erfahrung. Dieser Einwand ist jedoch bei der von uns vorgeschlagenen Vorgehensweise nicht so gravierend, da durch den Aufbau des Experimentes versucht werden kann, die Möglichkeit einer Wissensänderung auszuschließen. Indem eine Wissensänderung wiederum Lernen voraussetzt, liegt es im Manipulationsbereich des Experimentators, die experimentelle Situation so zu gestalten, daß eine Person keine Erfahrungen macht, welche dem als gelernt angenommenen Wissen widersprechen.

Den Verzicht auch als Handlungsmöglichkeit auszuzeichnen und als Handlungsalternative im Experiment zu verwenden, wirft keine neuen 
Probleme auf, da die Erwartung, dah der Verzicht auf ein Ziel $\mathrm{z}_{0}$ für das Eintreten eines Ereignisses $\mathrm{c}$ hinreichend ist, in der Definition D-3 über das Wissen eingeführt wurde.

\section{Diskussion}

Faßt man die dargestellten Überlegungen zusammen, so kommt man zu der Feststellung, daß die Aggressionsmaschine tatsächlich ein geeignetes Instrument ist, um die Klassifikationsprobleme in der experimentellen Aggressionsforschung zu überwinden. Aus dem vorgeschlagenen Klassifikationssystem können eindeutige Handlungsanweisungen für die Gestaltung von Versuchsanordnungen in der Aggressionsmaschine hergeleitet werden. Die zu stellenden Minimalforderungen an derartige Versuchsanordnungen sind:

1. die Verfügbarkeit mindestens einer ,nicht aggressiven" Handlungsalternative, und

2. die Herstellung und Kontrolle des relevanten Wissens der Versuchspersonen über die bestehenden Handlungsalternativen und deren Wirkungen.

Eine Sprachnormierung, wie von WERBIK (1971, 1974) verlangt, ist dabei nicht erforderlich. Für die Klassifikation der Handlung reicht es aus, auf den faktischen Sprachgebrauch bzw. auf das experimentell kontrollierte Wissen der Probanden zurückzugreifen. Eine Selbstklassifikation der Handlungen durch die Probanden erübrigt sich.

Die Brauchbarkeit des Klassifikationssystems ist aber nicht an die Aggressionsmaschine gebunden, sondern auch für andere, z. B. spieltheoretische, Versuchsanordnungen gegeben. Als Beispiel diene das ,,prisoner's dilemma game", in dem die Verfolgung der kompetitiven Strategie negativ intendiertes Handeln beinhaltet, während die Verfolgung der kooperativen Strategie als nicht negativ intendiert $z u$ klassifizieren ist.

Verfolgen zwei Spieler im ,prisoner's dilemma game" instruktionsgemäß das Ziel der Gewinnmaximierung und haben sie die Spielregeln verstanden, so impliziert die Wahl der kompetitiven Strategie die Erwartung, dał der Gegenspieler kooperativ spielen wird. Aufgrund des
Wissens über die Spielregeln beinhaltet die Wahl der kompetitiven Strategie dann stets die Erwartung, daß der Gegenspieler verlieren wird. Um die Eindeutigkeit der Klassifikation der kompetitiven Strategie zu garantieren, braucht also lediglich Wissen darüber sichergestellt zu werden, daß Verlust für den Gegenspieler unangenehm ist. Sowohl aufgrund der weitgehend gegebenen Einheitlichkeit des Alltagssprachgebrauchs des Terminus ,unangenehm" als auch im Sinne der Definition yon WERBIK $(1974$, p. 145 ff. $)$, kann dies als gesichert gelten, wenn der Spieler über die Zielsetzung des Gegenspielers, seinen Gewinn zu maximieren, Bescheid weiß. Die zu fordernden Voraussetzungen für die Klassifikation bestehen also lediglich darin, dass das Spiel von beiden Spielern ernsthaft gespielt wird.

In unserer Explikation des ,,prisoner's dilemma game" sind wir davon ausgegangen, daß die Auszahlungsmatrix des Spieles den Spielern bekannt ist, das Wissen über die Wirkungen des Spielverhaltens also mit den Spielregeln vermittelt wurde.

Grundsätzlich lassen sich drei Arten des Wissenserwerbs unterscheiden:

1. durch vorexperimentelle Erfahrung,

2. durch Instruktion und

3. durch während des Experimentes gewonnene Erfahrung.

Während man im zweiten Fall lediglich voraussetzen muß, daß3 die Beispiele und Erläuterungen des Versuchsleiters für die Ausbildung des entsprechenden Wissens der Probanden ausreichen, verlangen die anderen beiden Fälle eine nachträgliche empirische Überprüfung des Wissens. Im Experiment kann dies in zweierlei Weise geschehen:

1. durch Exploration der Probanden und

2. durch Herstellung experimenteller Situationen, in denen eine eindeutige Zuordnung zwischen der Wahl bestimmter Handlungsalternativen und dem relevanten Wissen besteht.

Für die Klassifikation von Alltagshandlungen treffen diese Möglichkeiten nur bedingt zu. Selbst wenn der eindeutige Nachweis, dass eine Person von bestimmten Wirkungszusammenhängen weiss, in der Alltagssituation nicht erbracht werden kann, so ist es mit Hilfe des Klassifika- 
tionssystems doch möglich zu explizieren, an welcher Stelle des Klassifikationsprozesses Deutungen stattfinden, nämlich bei den Annahmen über das Wissen der Person. Erst dadurch aber wird eine Problematisierung der auf Deutung beruhenden Klassifikation von Alltagshandlungen möglich.

\section{Literatur}

Buss, A.H. 1961. The psychology of Aggression. New York: Wiley.

Buss, A. H. 1971. Aggression Pays. In: Singer, J.L. (Ed.): The Control of Aggression and Violence. Cognitive and Physiological Factors. New York: Academic Press.

Buss, A. H. 1972. Die Quittung der Aggression. In: Singer, J. L. (Ed.): Steuerung von Aggression und Gewalt. Kognitive und physiologische Faktoren. Frankfurt/M: AVA.

DANN, H.-D. 1972. Aggression und Leistung. Stuttgart: Klett.

HIL KE, R. \& KEMPF, W.F. 1973. Kann Aggressionsforschung objektiv sein? Zum Problem der objektiven Klassifikation aggressiver Handlungen. Forschungsbericht 39 des Sonderforschungsbereiches 22 . Nürnberg: Sozialwissenschaftliches Forschungszentrum.

HiLKE, R., KeMPF, W. F. \& HÖll baChER, M. 1975. Die Messung nicht-provozierter Aggressivität. Psychol. Beiträge, 17, 371-391.

KEMPF, W.F. 1973. Eine kognitive Theorie, ,aggressiven" Handelns und Probleme ihrer experimentellen Realisation. III. Realisationsprobleme. Forschungsbericht 38 des Sonderforschungsbereiches 22 . Nürnberg: Sozialwissenschaftliches Forschungszentrum.
KEMPF, W.F. 1974. Basisprobleme der Diagnostik der Aggressivität. In: Eckensberger, L. (Ed.): Bericht über den 28. Kongress der Deutschen Gesellschaft für Psychologie in Saarbrücken 1972. Göttingen: Hogrefe.

MEAD, M. 1965. Leben in der Südsee. Jugend und Sexualität in primitiven Gesellschaften. München: Szczesny.

Schотт, F. 1971. Was ist Aggression? Ein Beitrag zum Problem der begrifflichen Eingrenzung und Anwendung psychologischer Konzepte. In: Selg, H. (Ed.): Zur Aggression verdammt? Stuttgart: Kohlhammer.

WerbiK, H. 1971. Das Problem der Definition ,aggressiver Verhaltensweisen. Zeitschrift für Sozialpsychologie 2, 233-247.

WERBiK, H. 1974. Theorie der Gewalt. München: Fink.

WERBIK, H. \& KEMPF, W. F. 1972. Eine kognitive Theorie ,,aggressiven" Handelns und Probleme ihrer experimentellen Realisierung. I. Aufbau einer kognitiven Terminologie. Forschungsbericht 16 des Sonderforschungsbereiches 22. Nürnberg: Sozialwissenschaftliches Forschungszentrum.

\section{Zu diesem Beitrag}

Diese Arbeit ist im Sonderforschungsbereich 22 ,Sozialisations- und Kommunikationsforschung", Universität Erlangen-Nürnberg, entstanden, der aus Mitteln der Deutschen Forschungsgemeinschaft finanziert wird.

Die Autoren danken Herrn Prof. Dr. Hans Werbik für wertvolle Anregung und Kritik. 